\title{
Quantum indirect estimation theory and joint estimate of all moments of two incompatible observables
}

\author{
G. M. D'Ariano, P. Perinotti, and M. F. Sacchi \\ QUIT Group, Dipartimento di Fisica "A. Volta", via Bassi 6, I-27100 Pavia, Italy and CNISM.
}

\begin{abstract}
We introduce the quantum indirect estimation theory, which provides a general framework to address the problem of which ensemble averages can be estimated by means of an available set of measuring apparatuses, e. g. estimate the ensemble average of an observable by measuring other observable. A main ingredient in this approach is that of informationally complete (infocomplete in short) measurements, which allow to estimate the ensemble average of any arbitrary system operator, as for quantum tomography. This naturally leads to the more stringent concept of $A B$ informationally complete measurements, by which one can estimate jointly all the moments of two incompatible observables $A$ and $B$. After analyzing all general properties of such measurements, we address the problem of their optimality, and we completely solve the case of qubits, showing that a $\sigma_{x} \sigma_{y}$-infocomplete measurement is less noisy than any infocomplete one. We will also discuss the relation between the concept of $A B$-infocompleteness and the notion of joint measurement of observables $A$ and $B$.
\end{abstract}

\section{INTRODUCTION}

The aim of any measurement is to retrieve information on the state of a physical system. In classical mechanics, measuring the location on the phase space provides a complete information on the system. On the other hand, in quantum mechanics there are infinitely many elementary measurements - corresponding to different observables - that provide only partial information, whereas "complementary" informations would require mutually exclusive experiments corresponding to non-commuting observables.

The problem then arises on how to perform a quantum measurement that can be used to infer information on non compatible observables. The idea is to make a generalized "unsharp" measurement [1], described by a socalled POVM (positive-operator valued measure), from which a specific type of information - such as e. a particular ensemble average of a given operator - is retrieved by a suitable data-processing of its experimental outcomes.

Of special interest are the informationally complete POVMs [2] —infocomplete POVMs in short - which span the whole operator space, thus allowing the estimation of arbitrary ensemble averages. Informationally complete measurements are relevant for foundations of quantum mechanics as a kind of "standard" for a purely probabilistic description [3]. Moreover, the existence of such measurements with minimal number of outcomes is crucial for the quantum version of the de Finetti theorem [4]. The most popular example of informationally complete measurement is given by the coherent-state POVM for a single mode of the radiation field, whose probability distribution is the so-called $Q$-function (or Husimi function) [5]. Another example, though of completely different kind, is the case of quantum tomography [6], in which one measures an observable randomly selected from an informationally complete set-a "quorum".

Investigations on informationally complete measurements have been extensively carried out. In the frame- work of "phase-space observables" [7, 8, ㅍ, 10, 11] the concept of informational completeness leads to substantial advancement on some relevant conceptual issues, such as the problem of jointly measuring non-commuting observables, or the problem of the classical limit of quantum measurements. A general classification of covariant infocomplete measurements has been given using grouptheoretical techniques [12], whereas the classification of the symmetric ones is still an open problem [13]. A thorough comparison of local with global infocomplete measurements for bipartite quantum systems has been carried out in Ref. [14]. On the other hand, for any general infocomplete measurement the optimal data-processing function for estimating the ensemble average of an arbitrary operator has been derived [15] with the help of frame theory [16, 17].

In this paper we introduce the quantum indirect estimation theory, which provides the general framework to address the problem of which ensemble averages can be estimated by means of an available set of measuring apparatuses. Typically, one has the problem of estimating the ensemble average of an observable by measuring other observables, or of estimating the expectation of a POVM - i. e. a probability distribution - by physically measuring another POVM. Essentially, one can estimate all expectations of operators that are linear combinations of POVM elements. The indirect estimation is achieved via a data processing of measurement outcomes. The data processing associates a numerical value to each outcome, depending on the ensemble average to be estimated. The final goal of the theory is then to optimize the data processing (generally not unique) in order to maximize statistical efficiency [15]. A special case of data-processing is the post-processing, which corresponds to probabilistic Boolean operations and permutations on the outcomes, with the data-processing function corresponding to a conditional probability. A typical example of post-processing is the coarse-graining of a POVM, in which each outcome is indeed a union of elementary outcomes, e. .g. in the marginalization of a bi-variate POVM. 
Clearly, a central role in quantum indirect estimation theory is played by infocomplete POVM's, by which one can estimate the ensemble averages of any arbitrary operator. However, for the estimation of the ensemble averages $\langle A\rangle$ and $\langle B\rangle$ of two (noncommuting) operators one does not necessarily need an infocomplete measurement, even in the case when one wants to estimate the full probability distribution of $A$ and $B$. In the last case one just needs a particular measurement, that we will introduce in the present paper, and which will be referred to as $A B$-infocomplete measurement. Indeed, the necessity of estimating complementary observables is the reason why the POVM which achieves the task is unsharp, and whence it adds noise to the POVM which can estimate a single observable. Likewise, one can infer that an $A B$ infocomplete POVM which is not infocomplete should add less noise than an infocomplete one, since the first kind of measurement avoids to collect redundant information. We will see that this indeed is true in the special case of qubits. We will also see that generally a joint measurement of observable $A$ and $B$ is not necessarily an $A B$-infocomplete measurement, whereas, viceversa, an $A B$-infocomplete measurement is an unbiased joint measurement of $A$ and $B$.

The paper is organized as follows. Sec. II is a long section where we introduce the quantum indirect estimation theory through the notion of partially informationally complete POVM, where the linear span of the POVM elements is a proper subspace of the Hilbert-Schmidt operator space. We also briefly review the theory of frames [16, 17], which generalize the concept of (operator) basis, and show how to characterize and optimize the processing functions of quantum measurements to estimate the expectation of observables. The notion of data-processing and post-processing are explained, and the concept of joint measurement of observables is recalled. In Sec. III minimal $A B$-infocomplete measurements are introduced, as the measurements described by POVMs whose span coincides with the span of $A, B$ and all their independent powers. A useful Lemma that gives sufficient conditions for minimality of the optimal $A B$-infocomplete measurement is proved. The case of qubits is solved in Sec. III.A, when the ensemble of unknown states corresponds to an isotropic distribution. Sec. IV is devoted to the conclusions.

\section{INDIRECT ESTIMATION THEORY}

A measurement on a quantum system 7] returns a random result $e$ from a set of possible outcomes $\mathrm{E}=\{e$ : $1, \ldots N\}$, with probability distribution $p(e \mid \rho)$ depending on the state $\rho$ of the system in a way which is distinctive of the measuring apparatus, according to the Born rule

$$
p(e \mid \rho)=\operatorname{Tr}\left[\rho P_{e}\right] .
$$

In Eq. (11) $P_{e}$ denote positive operators on the Hilbert space $\mathrm{H}$ of the system, representing our knowledge of the measuring apparatus from which we infer information on the state $\rho$ from the probability distribution $p(e \mid \rho)$. Positivity of $P_{e}$ is needed for positivity of $p(e \mid \rho)$, whereas normalization is guaranteed by the completeness relation $\sum_{e \in \mathrm{E}} P_{e}=I$. In the present paper we will only consider the simple case of finite discrete set E. More generally, one has an infinite probability space $\mathrm{E}$ (generally continuous), and in this context the set of positive operators $\left\{P_{e}\right\}$ becomes actually a positive operator valued measure (POVM), but we will keep the same acronym also for the discrete case, as usual in the literature. Every apparatus is described by a POVM, and, reversely, every POVM can be realized in principle by an apparatus [7, [8, [9]. Throughout this paper we will consider a quantum system with Hilbert space $\mathrm{H}$ with finite dimension $d=\operatorname{dim}(\mathrm{H})<+\infty$.

In the following we define the data processing $c_{i}^{X}$ for a POVM in order to reconstruct the ensemble average $\langle X\rangle$ of an operator $X \in \mathcal{L}(\mathrm{H})\left(c^{X}: i \mapsto c_{i}^{X}\right.$ is the so-called processing function).

\section{A. Informationally complete measurements}

We recall that the space of Hilbert-Schmidt operators is isomorphic to $\mathrm{H}^{\otimes 2}$, and coincides with the space $\mathcal{L}(\mathrm{H})$ of linear operators on $\mathrm{H}$ for finite dimensional Hilbert space $\mathrm{H} \sim \mathbb{C}^{d}$.

A POVM $\mathbf{P}$ is called informationally complete [2] if it linearly spans the whole operator space $\mathcal{L}(\mathrm{H})$. We generalize this concept to the following notion of partially informationally complete POVM

Definition 1 For $\mathcal{R}$ a linear operator space, we will call a POVM $\mathcal{R}$-informationally complete, if $\mathcal{R} \subseteq \operatorname{Span}(\mathbf{P})$.

We have used the natural notation $\operatorname{Span}(\mathbf{P})=$ $\operatorname{Span}\left(P_{1}, P_{2}, \ldots P_{N}\right) \in \mathcal{L}(\mathrm{H})$. The projection on the linear operator space $\mathcal{R}$ will be denoted by $\Pi_{\mathcal{R}}$.

It is clear that the knowledge of probabilities of an $\mathcal{R}$ informationally complete POVM allows the calculation of ensemble averages $\langle X\rangle_{\rho}$ for all $X \in \mathcal{R}$ by the simple formula

$$
\langle X\rangle_{\rho}=\sum_{i=1}^{N} c_{i}^{X} \operatorname{Tr}\left[\rho P_{i}\right] .
$$

$c_{i}^{X}$ denoting the data processing for $X$. Eq. (2) has to be regarded as the definition itself of the processing function $c^{X}$, in the sense that the coefficients $c_{i}^{X}$ must satisfy Eq. (2) as a constraint. If the POVM elements are linearly independent then the processing function $c^{X}: i \mapsto c_{i}^{X}$ for an operator $X$ is unique, whereas for linearly dependent POVM elements the possible choices are infinite (notice that even thorough Eq. (2) explicitly contains the processing function, its value is independent of the specific choice of $c_{i}^{X}$ ). These facts determine two questions: a) how to find a suitable processing function $c^{X}$ for a given 
operator $X$; b) which is the processing function $c^{X}$ minimizing the statistical error

$$
\delta_{\rho}^{2}(X)=\sum_{i=1}^{N}\left|c_{i}^{X}\right|^{2} \operatorname{Tr}\left[\rho P_{i}\right]-\langle X\rangle_{\rho}^{2},
$$

where, for simplicity, we restrict to selfadjoint $X$ (notice that the actual statistical error is obtained by dividing $\delta_{\rho}(X)$ by $\sqrt{N_{e x}-1}$, with $N_{e x}$ the number of experiments). In order to answer these questions we will consider some elementary results in frame theory.

\section{B. Elements of frame theory}

A frame in a Hilbert space K [16, 17] (for the sake of simplicity we will consider finite dimensional spaces) is a set of vectors $\left\{v_{i}\right\}_{1 \leq i \leq N} \subseteq \mathrm{K}$, with $N \leq \infty$ such that there exist two constants $0<a \leq b<\infty$ and

$$
a\|\psi\|_{\mathrm{K}}^{2} \leq \sum_{i=1}^{N}\left|\left\langle v_{i} \mid \psi\right\rangle\right|^{2} \leq b\|\psi\|_{\mathrm{K}}^{2},
$$

and one can prove that for finite dimensional systems the property of a set $\left\{v_{i}\right\}$ of being a frame is equivalent to completeness, namely for all $\psi \in \mathrm{K}$ one can expand $\psi$ on the vectors $\left\{v_{i}\right\}$ by suitable coefficients. On the other hand, given a set of vectors $\left\{v_{i}\right\}$ on $\mathrm{K}$ they are a frame iff the frame operator

$$
F=\sum_{i=1}^{N}\left|v_{i}\right\rangle\left\langle v_{i}\right|
$$

is bounded and invertible. In this case, defining the canonical dual frame $\left\{w_{i}\right\}$ by $F^{-1}\left|v_{i}\right\rangle=\left|w_{i}\right\rangle$ one has

$$
F F^{-1}=\sum_{i=1}^{N}\left|v_{i}\right\rangle\left\langle w_{i}\right|=I,
$$

and clearly the coefficients $\left\langle w_{i} \mid \psi\right\rangle$ are suitable for the expansion of $\psi$ on the frame $\left\{v_{i}\right\}$, namely

$$
|\psi\rangle=\sum_{i=1}^{N}\left|v_{i}\right\rangle\left\langle w_{i} \mid \psi\right\rangle
$$

The second interesting result [18] is the following classification of all possible alternate dual frames $\left\{z_{i}\right\}$ such that $\sum_{i=1}^{N}\left|v_{i}\right\rangle\left\langle z_{i}\right|=I$, which are given by

$$
\left|z_{i}\right\rangle=\left|w_{i}\right\rangle+\left|y_{i}\right\rangle-\sum_{j=1}^{N}\left|y_{j}\right\rangle\left\langle v_{j} \mid w_{i}\right\rangle,
$$

where $\left\{y_{i}\right\} \subseteq \mathrm{K}$ is arbitrary. If we now consider the POVM $\mathbf{P}$ and $K \equiv \operatorname{Span}(\mathbf{P}) \subseteq \mathcal{L}(\mathrm{H})$, clearly the POVM elements are a frame for $\operatorname{Span}(\mathbf{P})$, and a suitable processing function $c^{X}$ for an operator $X$ is provided by the canonical dual frame. This answers the first question about finding processing functions. In the next section we will use the classification of alternate duals in Eq. (8) to answer the second question about the minimization of the statistical error.

\section{Optimization of the processing function}

The quantity we want to minimize is the statistical error in Eq. (3). Since the processing function is involved only in the first term, the quantity to be minimized is the following

$$
\delta_{\rho}^{2}(X)+\langle X\rangle_{\rho}^{2}=\sum_{i=1}^{N}\left|c_{i}^{X}\right|^{2} \operatorname{Tr}\left[\rho P_{i}\right] .
$$

This quantity depends on the state $\rho$, but in a Bayesian framework we can make it independent of $\rho$ by suitably averaging Eq. (3) over a prior ensemble $\mathcal{E}=$ $\left\{q_{j}, \rho_{j}\right\}_{1 \leq j \leq M}$, obtaining

$$
\delta_{\mathcal{E}}^{2}(X)=\sum_{i=1}^{N}\left|c_{i}^{X}\right|^{2} \operatorname{Tr}\left[\rho_{\mathcal{E}} P_{i}\right]-\overline{\langle X\rangle^{2}}{ }_{\mathcal{E}}=\sum_{j=1}^{M} q_{j} \delta_{\rho_{j}}^{2}(X)
$$

where $\rho_{\mathcal{E}}:=\sum_{j=1}^{M} q_{j} \rho_{j}$, and $\overline{\langle X\rangle^{2}}:=\sum_{j=1}^{M} q_{j}\langle X\rangle_{\rho_{j}}^{2}$. The only term depending on the processing function is $\sum_{i=1}^{N}\left|c_{i}^{X}\right|^{2} \operatorname{Tr}\left[\rho_{\mathcal{E}} P_{i}\right]$, which can be viewed as a norm for the vector $c^{X}$ of coefficients in a Euclidean space $\mathrm{K}$, where the metric matrix $\pi$ is diagonal on the canonical basis and has eigenvalues $\pi_{i i}=\operatorname{Tr}\left[\rho_{\mathcal{E}} P_{i}\right]$. We can now define the linear operator $\Lambda: \mathrm{K} \rightarrow \operatorname{Span}(\mathbf{P})$ such that

$$
\Lambda c=\sum_{i=1}^{N} c_{i} P_{i}
$$

which has the following matrix elements $\Lambda_{m n, i}=\left(P_{i}\right)_{m n}$, and all the generalized inverses $\Gamma: \operatorname{Span}(\mathbf{P}) \rightarrow \mathrm{K}$ of $\Lambda$ satisfying $\Lambda \Gamma \Lambda=\Lambda$ are in correspondence with alternate duals $\mathbf{D}$ by the identity $\Gamma_{i, m n}=\left(D_{i}^{*}\right)_{m n}$. Generalizing the proof for the minimum norm pseudoinverse in Ref. [19] it was proved in Ref. [14] that the minimum norm is achieved by $\Gamma$ satisfying

$$
\pi \Gamma \Lambda=\Lambda^{\dagger} \Gamma^{\dagger} \pi
$$

and the corresponding optimal dual was derived in Ref. [15], and can be expressed as follows

$$
D_{i}=\Delta_{i}-\sum_{j=1}^{N}\left\{[(I-M) \pi(I-M)]^{\ddagger} \pi\right\}_{i j} \Delta_{j},
$$

where $\left\{\Delta_{i}\right\}$ is the canonical dual and the projection matrix $M$ has matrix elements $M_{i j}=\operatorname{Tr}\left[\Delta_{i} P_{j}\right]$. The symbol $Y^{\ddagger}$ denotes the Moore-Penrose generalized inverse of $Y$, namely the symmetric, minimum norm and least 
squares generalized inverse $Z$ satisfying the conditions: $Z Y Z=Z, Z Y=Y^{\dagger} Z^{\dagger}, Y Z=Z^{\dagger} Y^{\dagger}$. In the following we will make use of the following compact formula for the minimum noise, which was derived in Ref. [20]

$$
\delta_{\mathcal{E}}^{2}(X)=\left\langle X\left|\left(\Lambda \pi^{-1} \Lambda^{\dagger}\right)^{-1}\right| X\right\rangle-{\overline{\langle X\rangle_{\mathcal{E}}}}
$$

where $|X\rangle \in \mathrm{H}^{\otimes 2}$ is the vector corresponding to $X$ as follows

$$
|X\rangle:=\sum_{m, n=1}^{d} X_{m n}|m\rangle \otimes|n\rangle \leftrightarrow X,
$$

for fixed basis $\{|m\rangle\}_{1 \leq m \leq d}$ in $\mathrm{H}$. The following identities are easily verified

$$
\langle X \mid Y\rangle=\operatorname{Tr}\left[X^{\dagger} Y\right], A \otimes B|X\rangle=\left|A X B^{T}\right\rangle, E|X\rangle=\left|X^{T}\right\rangle
$$

where $X^{T}$ is the transpose of $X$ in the canonical basis, and $E$ is the swap operator $E|\phi\rangle \otimes|\psi\rangle=|\psi\rangle \otimes|\phi\rangle$. Throughout the paper we will use the following notation for orthogonal projectors over Hilbert-Schmidt subspaces $\mathcal{S} \subseteq \mathcal{L}(\mathrm{H})$

$\Pi_{\mathcal{S}}:=$ orthogonal projector over $\operatorname{Span}\{|X\rangle, X \in \mathcal{S}\}$.

Since the POVM $\mathbf{P}$ is selfadjoint, namely $E\left|P_{i}^{*}\right\rangle=\left|P_{i}\right\rangle$ $\left(F^{*}=\left(F^{\dagger}\right)^{T}\right.$ denotes the complex conjugated operator $)$, its frame operator $F=\sum_{i=1}^{N}\left|P_{i}\right\rangle\left\langle P_{i}\right|$ enjoys the following property

$$
E F^{*} E=F
$$

which is clearly shared by its inverse and by $\Pi_{\mathcal{S}}=F^{-1} F$. The canonical dual $\left\{\Delta_{i}\right\}$ satisfies then the following identity

$$
E\left|\Delta_{i}^{*}\right\rangle=E F^{-1 *}\left|P_{i}^{*}\right\rangle=F^{-1} E\left|P_{i}^{*}\right\rangle=F^{-1}\left|P_{i}\right\rangle=\left|\Delta_{i}\right\rangle,
$$

namely $\Delta_{i}^{\dagger}=\Delta_{i}$. Since all alternate duals $\mathbf{D}$ satisfy

$$
\sum_{i=1}^{N}\left|D_{i}\right\rangle\left\langle P_{i}\left|=\Pi_{\mathrm{Span}(\mathbf{P})}=E \Pi_{\mathrm{Span}(\mathbf{P})}^{*} E=\sum_{i=1}^{N}\right| D_{i}^{\dagger}\right\rangle\left\langle P_{i}\right|,
$$

it is clear that if $\mathbf{D}$ is an alternate dual then also $\mathbf{D}^{\dagger}$ is. It is easy to verify that also $1 / 2\left(D_{i}+D_{i}^{\dagger}\right)$ is an alternate dual. Suppose now that the optimal dual is not selfadjoint, then there exists a selfadjoint $X$ such that $\Im\left(\operatorname{Tr}\left[D_{i}^{\dagger} X\right]\right) \neq 0$, and the minimum statistical error for $X$ would be

$$
\begin{aligned}
\delta_{\mathcal{E}}^{2}(X)= & \sum_{i=1}^{N}\left|\operatorname{Tr}\left[D_{i}^{\dagger} X\right]\right|^{2} \operatorname{Tr}\left[\rho_{\mathcal{E}} P_{i}\right]-{\overline{\langle X\rangle_{\mathcal{E}}}}> \\
& \sum_{i=1}^{N} \Re\left(\operatorname{Tr}\left[D_{i}^{\dagger} X\right]\right)^{2} \operatorname{Tr}\left[\rho_{\mathcal{E}} P_{i}\right]-{\overline{\langle X\rangle_{\mathcal{E}}}}^{N}= \\
& \sum_{i=1}^{N}\left(\operatorname{Tr}\left[\left(D_{i}^{\dagger}+D_{i}\right) X\right] / 2\right)^{2} \operatorname{Tr}\left[\rho_{\mathcal{E}} P_{i}\right]-\overline{\langle X\rangle}_{\mathcal{E}}
\end{aligned}
$$

This is clearly absurd, since the last line is the statistical error given by the dual $\left(D_{i}+D_{i}^{\dagger}\right) / 2$. The canonical dual and the optimal dual for any ensemble are then selfadjoint.

Writing the matrix elements of both sides in Eq. (12), and considering that $\Gamma \Lambda_{i j}=\operatorname{Tr}\left[D_{i}^{\dagger} P_{j}\right]$, one has $\pi_{i i} \operatorname{Tr}\left[D_{i}^{\dagger} P_{j}\right]=\operatorname{Tr}\left[P_{i} D_{j}\right] \pi_{j j}$. Summing both sides over the index $i$ we obtain $\operatorname{Tr}\left[\rho_{\mathcal{E}} P_{j}\right]=\operatorname{Tr}\left[D_{j}\right] \operatorname{Tr}\left[\rho_{\mathcal{E}} P_{j}\right]$, and consequently $\operatorname{Tr}\left[D_{i}\right]=1$ for all $i$ such that $\operatorname{Tr}\left[P_{i} \rho_{\mathcal{E}}\right] \neq 0$.

\section{Post-processing}

We will call post-processing of a POVM a dataprocessing which maps the POVM into another POVM, namely

$$
Q_{j}=\sum_{i=1}^{N} m(j \mid i) P_{i}
$$

where $m(j \mid i)$ is a conditional probability, namely the corresponding matrix is Markov, i. e. $m(j \mid i) \geq 0$ and $\sum_{j} m(j \mid i)=1 \forall i$. Clearly the post-processing is a special case of data-processing array, corresponding to

$$
c_{i}^{Q_{j}} \equiv m(j \mid i)
$$

Even though it can be regarded as a special case of dataprocessing, the post-processing is conceptually very different, being the randomization of set-theoretical operations. Indeed, it corresponds to a randomization of the following operations

T1 identification of two outcomes, e. g. $j$ and $k$ are identified with the same outcome $l$, corresponding to $m(n \mid j)=\delta_{l n}$ and $m(n \mid k)=\delta_{l n}$;

T2 permutation $\pi$ of outcomes, corresponding to $m(\pi(j) \mid k)=\delta_{j k}$

T3 splitting of one outcome $l$ into two outcomes $j$ and $k$, corresponding to choosing $j$ with probability $m(j \mid l)=p$ and $k$ with probability $m(k \mid l)=1-p$, $0<p<1$.

We can see that generally the cardinality of $\mathbf{Q}$ is different from that of $\mathbf{P}$. Also, notice that a data processing array $c_{i}^{Q_{j}}$ for the POVM $\mathbf{Q}$ is not necessarily a Markov matrix, since generally $c_{i}^{Q_{j}} \nsucceq 0$, and also one not necessarily has normalization $\sum_{j} c_{i}^{Q_{j}}=1 \forall i$, due to linear dependence of the POVM $\mathbf{P}$, even though, there always exists an alternate data processing that is normalized.

When two POVMs $\mathbf{P}$ and $\mathbf{Q}$ are connected by post-processing we will write $\mathbf{P} \succ \mathbf{Q}$, and say that the POVM $\mathbf{P}$ is cleaner under post-processing-postprocessing cleaner in short-than the POVM Q. The relation $\succ$ is a pseudo-ordering, since it is $i$ ) reflexive, corresponding to $\left.\mathbf{P} \succ \mathbf{P}, m(i \mid j)=\delta_{i j} ; \quad i i\right)$ 
transitive, i. e. $\mathbf{P} \succ \mathbf{Q} \succ \mathbf{R}$, corresponding to $R_{i}=\sum_{j} m(i \mid k) Q_{k}, \quad Q_{k}=\sum_{j} m^{\prime}(k \mid j) P_{j}, \Rightarrow R_{i}=$ $\sum_{j} m^{\prime \prime}(i \mid j) P_{j}, m^{\prime \prime}(i \mid j)=\sum_{k} m(i \mid k) m^{\prime}(k \mid j)$.

We can define a partial ordering and an equivalence relation in terms of the POVM post-processing as follows.

Definition 2 The POVM's $\mathbf{P}$ and $\mathbf{Q}$ are postprocessing equivalent - in symbols $\mathbf{P} \simeq \mathbf{Q}$-iff both relations $\mathbf{P} \succ \mathbf{Q}$ and $\mathbf{Q} \succ \mathbf{P}$ hold.

We are now in position to define cleanness under post processing, namely

Definition 3 A POVM $\mathbf{P}$ is post-processing clean if for any POVM $\mathbf{Q}$ such that $\mathbf{Q} \succ \mathbf{P}$, then also $\mathbf{P} \succ \mathbf{Q}$ holds, namely $\mathbf{P} \simeq \mathbf{Q}$.

The characterization of cleanness under post-processing is very simple, and is given by the following theorem.

Theorem 1 [21] A POVM $\mathbf{P}$ is post-processing clean iff it is rank-one.

We address the reader to Ref. [21] for the proof of the Theorem.

For a POVM $\mathbf{Q}$ with $\mathbf{Q} \nprec \mathbf{P}$ i. e. which is not a post-processing of $\mathbf{P}$ one can anyway introduce another smeared-out version $\tilde{\mathbf{Q}}$ of $\mathbf{Q}$

$$
\tilde{Q}_{j}:=\frac{Q_{j}+\alpha_{j} I}{1+\sum_{l} \alpha_{l}}, \quad \alpha_{j}=\max _{i}\left\{0,-c_{i}^{Q_{j}}\right\}
$$

such that $\tilde{\mathbf{Q}} \prec \mathbf{P}-$ i. e. $\tilde{\mathbf{Q}}$ is a post-processing of $\mathbf{P}$. The Markov matrix is simply given by

$$
m(j \mid i)=\frac{c_{i}^{Q_{j}}+\alpha_{j}}{1+\sum_{l} \alpha_{l}} .
$$

The perfect measurement of an observable corresponds to a POVM made with the orthogonal projectors $X_{j}$ over its eigenspaces, and we will write $\mathbf{X}=\left[X_{j}\right]$ with

$$
X_{j} X_{i}=\delta_{i j} X_{i} \geq 0, \quad \sum_{i} X_{i}=I .
$$

More generally, we will say that

Definition 4 A POVM $\mathbf{P}$ describes an imperfect measurement of the observable $\mathbf{X}$ if $\mathbf{X} \succ \mathbf{P}$, namely the POVM $\mathbf{P}$ is a post-processing of $\mathbf{X}$.

In practical terms this means that the measurement is a smearing-out of the perfect observable due to additional noise which is ascribed to the output stage of the measuring apparatus. One can see that mathematically a POVM is a measurement of the observable $\mathbf{X}$ when it commutes with the observable. In this way the POVM $\mathbf{P}$ describing an imperfect measurement of $X$ will be simply a function $P_{i}=P_{i}(X)$ of the operator $X$.
The concept of post-processing allows to introduce a general notion of joint measurement of (generally non commuting) observables.

Definition 5 (Joint measurement of observables) We say that a POVM $\mathbf{P}$ achieves the joint measurement of the observables $\mathbf{X}^{(1)}, \mathbf{X}^{(2)}$,..., if for every observable $\mathbf{X}^{(i)}$ of the list there is a post-processing of $\mathbf{P}$ which achieves an imperfect measurement of $\mathbf{X}^{(i)}$.

We stress that in our operational point of view it is irrelevant that a joint measurement is described by a bivariate probability distribution (which could be interpreted in terms of the alleged outcomes of the non commuting observables $A$ and $B$ ). The only thing that matters is the possibility of performing jointly imperfect measurements of both $A$ and $B$, since, indeed, the joint probability of their eigenvalues is counterfactual.

The present definition of joint measurement for different observables is sufficiently comprehensive to include all known joint measurements, such as the joint measurement of position and momentum [22], and the measurement of the direction of an angular momentum, corresponding to a POVM made with spin-coherent states [23]. Indeed, the usual definition of joint measurement simply involves the marginalization of multivariate POVMs. A natural generalization of such definition of joint measurement for non multivariate POVM's would be simply to consider the marginalization as the identification of outcomes in T1. Our definition of joint measurements further generalizes the notion to any postprocessing, introducing also the natural transformations T2 and T3.

We should notice that our definition (as the standard ones) of joint measurements also includes some trivial cases, in particular: a) pure guessing post-processing, with Markov matrix with equal columns (data processing independent of the outcome), corresponding to a smeared-out POVM having each element proportional to the identity (clearly for such trivial smearing-out each POVM is the joint measurement of any set of observables); b) the POVM $\mathbf{P}$ achieving the joint measurement is actually the random selection of one observable at a time, namely $\mathbf{P}=\cup_{i} \lambda_{i} \mathbf{X}^{(i)}$, where we define the convex union $\mathbf{R}=\lambda \mathbf{P} \cup(1-\lambda) \mathbf{Q}$ of two POVMs $\mathbf{P}$ and $\mathbf{Q}$ with cardinalities $|\mathbf{P}|=N$ and $|\mathbf{Q}|=M$ as follows

$$
\begin{aligned}
& \mathbf{R}=\lambda \mathbf{P} \cup(1-\lambda) \mathbf{Q}:= \\
& {\left[\lambda P_{1}, \ldots, \lambda P_{N},(1-\lambda) Q_{1}, \ldots,(1-\lambda) Q_{M}\right]}
\end{aligned}
$$

(more generally one can have even the random selection of imperfect measurements of noncommuting observables). In the following we will call the above joint measurements trivial. 


\section{E. Measuring a POVM by another POVM}

A special case of processing is the one corresponding to another POVM $\mathbf{Q}=\left(Q_{1}, Q_{2}, \ldots, Q_{M}\right)$ in the span $\operatorname{Span}(\mathbf{P})$. Notice that, even though one has the linearity of processing functions $c_{i}^{X+Y}=c_{i}^{X}+c_{i}^{Y}$, for linearly dependent POVM $\mathbf{P}$ the processing function is not unique, whence, generally $c_{i}^{I} \neq 1$, which implies that the processing function $c_{i}^{Q_{j}}$ for the POVM elements $Q_{j}$ generally do not satisfy the normalization condition $\sum_{j=1}^{M} c_{i}^{Q_{j}}=1$. In addition, generally for $X \geq 0$ not necessarily one has $c_{i}^{X} \geq 0$. This implies that $c_{i}^{Q_{j}}$ cannot be treated as conditional probabilities $p(j \mid i):=c_{i}^{Q_{j}}$. Therefore, it is not generally true that a POVM $\mathbf{Q} \in \operatorname{Span}(\mathbf{P})$ can be achieved as a post-processing of $\mathbf{P}$. However, even though $\mathbf{Q}$ cannot be obtained in this way, this is possible for a blurred version of it according to the following theorem

Theorem 2 Given a POVM $\mathbf{Q} \in \operatorname{Span}(\mathbf{P})$, there exists a POVM $\mathbf{Q}^{\prime} \prec \mathbf{Q}$ that is a post-processing of $\mathbf{P}$, or, in other words, $\mathbf{Q}^{\prime} \prec \mathbf{Q}$ and $\mathbf{Q}^{\prime} \prec \mathbf{P}$.

Proof. As shown at the end of Sec. II C, the normalization requirement is satisfied at least by the optimal processing, since $c_{i}^{I}=\operatorname{Tr}\left[D_{i} I\right]=1$ for all $i$, for the optimal dual $\mathbf{D}$ of $\mathbf{P}$. For $c_{i}^{Q_{j}} \nsupseteq 0$, we can consider the "blurred" $\operatorname{POVM} \mathbf{Q}(\varepsilon)$ with $Q_{i}(\varepsilon)=(1-\varepsilon) Q_{i}+\varepsilon \frac{I}{M}$, which, for sufficiently large $\varepsilon>0$ has $c_{i}^{Q_{j}(\varepsilon)} \geq 0$. The minimum value of $\varepsilon$ is $\varepsilon_{*}=-\frac{M \bar{c}}{1-M \bar{c}}$, where $\bar{c}=\min \left\{0, \min _{i, j}\left\{c_{i}^{Q_{j}}\right\}\right\}$.

How can we interpret the indirect measurement of $\mathbf{Q}$ ? In our approach to the theory of statistics of quantum measurements the POVM represents a question asked by the experimenter, and the answer is the outcome. A POVM Q in the space $\operatorname{Span}(\mathbf{P})$ associated to the POVM $\mathbf{P}$ is a question that can be indirectly asked through the POVM $\mathbf{P}$, corresponding to the following rule: for given outcome $i$ of the POVM $\mathbf{P}$ pick the answer $j$ out of the set $1, \ldots M$ randomly according to the conditional probability $p(j \mid i)=c_{i}^{Q_{j}\left(\varepsilon_{*}\right)}$.

If we collect the statistics for the answers $j$ obtained through this strategy, we asymptotically obtain the probabilities

$$
\operatorname{Tr}\left[\rho Q_{j}\left(\varepsilon_{*}\right)\right]=\left(1-\varepsilon_{*}\right) \operatorname{Tr}\left[\rho Q_{j}\right]+\frac{\varepsilon_{*}}{M} .
$$

The estimated probabilities are not exactly $\operatorname{Tr}\left[\rho Q_{j}\right]$, but since $\varepsilon_{*}$ is exactly known, one can retrieve $\operatorname{Tr}\left[\rho Q_{j}\right]$ by the formula

$$
\operatorname{Tr}\left[\rho Q_{j}\right]=\frac{1}{1-\varepsilon_{*}}\left(\operatorname{Tr}\left[\rho Q_{j}\left(\varepsilon_{*}\right)\right]-\frac{\varepsilon_{*}}{M}\right) .
$$

The statistical error on such estimate of $\operatorname{Tr}\left[\rho Q_{j}\right]$ is now given by $\sum_{i=1}^{N}\left|c_{i}^{Q_{j}}-\operatorname{Tr}\left[\rho Q_{j}\right]\right|^{2} \operatorname{Tr}\left[\rho_{\mathcal{E}} P_{i}\right]$, and since

$$
c_{i}^{Q_{j}}=\frac{1}{1-\varepsilon_{*}}\left(c_{i}^{Q_{j}\left(\varepsilon_{*}\right)}-\frac{\varepsilon_{*}}{M}\right),
$$

the statistical error in the estimate of the probabilities $\operatorname{Tr}\left[\rho Q_{j}\right]$ is just $\frac{1}{\left(1-\varepsilon_{*}\right)^{2}}$ times greater than the statistical error in the estimate of $\operatorname{Tr}\left[\rho Q_{j}\left(\varepsilon_{*}\right)\right]$, and the estimated probability $\operatorname{Tr}\left[\rho Q_{j}\right]$ is unbiased.

Moreover, if the POVM $\mathbf{Q}$ is the spectral decomposition of an operator $X$, then one can obtain $\langle X\rangle$ by taking $\sum_{j=1}^{M} x_{j}\left\langle Q_{j}\right\rangle$. The minimum error in the estimate is the same that one would obtain by estimating $\left\langle X\left(\varepsilon_{*}\right)\right\rangle=$ $\left(1-\varepsilon_{*}\right)\langle X\rangle+\operatorname{Tr}[X] \varepsilon_{*} / M$, where $X\left(\varepsilon_{*}\right)=\sum_{j=1}^{M} Q_{j}^{\prime} \lambda_{j}$, and then calculating $\langle X\rangle$ by taking

$$
\langle X\rangle=\frac{1}{\left(1-\varepsilon_{*}\right)}\left(\left\langle X\left(\varepsilon_{*}\right)\right\rangle-\frac{\varepsilon_{*}}{M} \operatorname{Tr}[X]\right) .
$$

Notice that the coefficients $c_{i}^{Q_{j}}$ can then be interpreted as matrix elements of a linear transformation that brings eigenvalues $x_{j}$ of $X$ to the processing function for $\mathrm{X}$ $c_{i}^{X}=\sum_{j=1}^{M} c_{i}^{Q_{j}} x_{j}$. If the $c_{i}^{Q_{j}}$ are evaluated through the optimal dual, we can say that $c_{i}^{X}$ is the best estimate of $X$ provided that the outcome $i$ has occurred in a measurement of the POVM $\mathbf{P}$, since the estimate of $\langle X\rangle$ rising from this strategy has the minimum statistical error.

From Theorem 7 and Definitions 1 and 6, it follows immediately that

Theorem 3 Every $\mathcal{R}$-informationally complete measurement is an an unbiased joint measurement of all observables in $\operatorname{Span}\left(\mathcal{R} \cup \mathcal{R}^{\dagger}\right)$,

where we denoted by $\mathcal{R}^{\dagger}$ the linear space spanned by the adjoints of all operators in $\mathcal{R}$. Moreover, one has

Corollary 1 Every informationally complete measurement is a nontrivial joint measurement of all observables.

\section{AB-INFORMATIONALLY COMPLETE MEASUREMENTS}

The problem of estimating the full probability distribution of two noncommuting observables $A$ and $B$ can be treated by considering the space spanned by independent powers of $A$ and $B$, which we call $A B$-space

$$
\mathcal{S}_{A B}=\operatorname{Span}\left\{A^{n}, B^{n}, n=0,1,2, \ldots\right\} .
$$

The corresponding projection (in the sense of Eq. (17)) will be denoted by $\Pi_{A B}$. The POVMs allowing for simultaneous measurement of $A, B$, and their independent powers are what we call $A B$-informationally complete measurements, whose space $\operatorname{Span}(\mathbf{P})$ contains $\mathcal{S}_{A B}$.

Usually in the literature, a self-adjoint operator $X=$ $\sum_{i} x_{i} X_{i}$ is associated to the observable $\mathbf{X}$, and the probability distribution $p(i \mid \rho)=\operatorname{Tr}\left[X_{i} \rho\right]$ is recovered by the moments of $X$ through the set of eigenvalues $x_{i} \in \mathbb{R}$. The relation between probabilities and moments passes 
through the identity

$$
X_{h}=\sum_{j=0}^{s-1} \mathbf{W}_{j h} X^{j}=\sum_{j=0}^{s-1} \sum_{k=1}^{s} \mathbf{W}_{j h} x_{k}^{j} X_{k}
$$

whence $\sum_{j=0}^{s-1} \mathbf{W}_{j h} x_{k}^{j}=\delta_{h k}$, namely $\mathbf{W}$ is the inverse of the Vandermonde matrix $\mathbf{W}^{-1}=\left\{x_{k}^{j}\right\}$. Linear independence of the first $s-1$ powers of $X$ (and linear dependence of any higher power), where $s$ is the cardinality of the spectrum of $X$, follows from the fact that the minimal polynomial of $X$

$$
m_{X}(x)=\prod_{h=1}^{s}\left(x-x_{h}\right)
$$

vanishes as $m_{X}(X)=0$, and it is the minimal degree polynomial vanishing at $X$, whence all powers $X^{n}, 0 \leq$ $n \leq s-1$, and only such powers, are linearly independent.

Using Theorem 2 we see that there always exist two data processing of an $A B$-infocomplete measurement giving two unbiased Abelian POVMs commuting with $A$ and $B$, respectively. Therefore, one has

Corollary 2 Every AB-informationally complete measurement is an unbiased joint measurement of observables $A^{n}$ and $B^{n}$, for all integer $n$.

A special case is that of minimal $A B$-informationally complete POVMs, whose space $\operatorname{Span}(\mathbf{P})$ exactly coincides with $\mathcal{S}_{A B}$. Notice that an example of $A B$ informationally complete POVM is readily given by the union of the two orthonormal resolutions of $A$ and $B$ with a rescaling by a factor $\frac{1}{2}$. From this example we can conclude that the projection $\Pi_{A B}$ also enjoys the property $E \Pi_{A B}^{*} E=\Pi_{A B}$. We can translate the two properties of simultaneous measurements and $A B$-informationally complete measurements as follows:

1. $\mathbf{P}$ is $A B$-informationally complete iff

$$
\Pi_{A B} \Pi_{\mathrm{Span}(\mathbf{P})}=\Pi_{\mathrm{Span}(\mathbf{P})} \Pi_{A B}=\Pi_{A B} .
$$

2. $\mathbf{P}$ is minimal $A B$-informationally complete iff

$$
\Pi_{\mathrm{Span}(\mathbf{P})} \equiv \Pi_{A B}
$$

Notice that a joint measurement of $A$ and $B$ is generally non minimal, e. g. it provides also estimation of correlations, which is the case of the joint measurement of position and momentum which minimizes the product of uncertainties [22], or of the covariant measurement of the angular momentum [23]. We conjecture that the minimum-error POVM's belong to the set of minimal $A B$-informationally complete POVMs. In the next session we will show that for $\rho_{\mathcal{E}} \in \mathcal{S}_{A B}$ the conjecture is true for dimension $d=2$. Moreover, we have the following
Lemma 1 Sufficient conditions for minimality of the optimal AB-informationally complete POVM $\mathbf{Q}$ :

1. the state $\rho_{\mathcal{E}}$ belongs to $\mathcal{S}_{A B}$;

2. there exists an optimal POVM $\mathbf{P}$ which is $A B$ informationally complete, and such that the operators $Q_{i}$ given by $\left|Q_{i}\right\rangle=\Pi_{A B}\left|P_{i}\right\rangle$ are all positive.

Proof. Let us consider the minimum error in Eq. (14), and recall that we are interested in operators $X$ such that $\Pi_{A B}|X\rangle=|X\rangle$. Then

$$
\delta_{\mathcal{E}}(X)=\left\langle X\left|\Pi_{A B}\left(\Lambda \pi^{-1} \Lambda^{\dagger}\right)^{-1} \Pi_{A B}\right| X\right\rangle-\overline{\langle X\rangle}_{\mathcal{E}} .
$$

Since 25] $\Pi_{A B}\left(\Lambda \pi^{-1} \Lambda^{\dagger}\right)^{-1} \Pi_{A B} \geq\left(\Pi_{A B} \Lambda \pi^{-1} \Lambda^{\dagger} \Pi_{A B}\right)^{-1}$, and since $\Lambda \pi^{-1} \Lambda^{\dagger}=\sum_{i=1}^{N} \frac{1}{\operatorname{Tr}\left[P_{i} \rho_{\mathcal{E}}\right]}\left|P_{i}\right\rangle\left\langle P_{i}\right|$, then we have to minimize

$$
\begin{aligned}
& \left\langle X\left|\left(\Pi_{A B} \Lambda \pi^{-1} \Lambda^{\dagger} \Pi_{A B}\right)^{-1}\right| X\right\rangle \\
= & \left\langle X\left|\left(\sum_{i=1}^{N} \frac{1}{\operatorname{Tr}\left[P_{i} \rho_{\mathcal{E}}\right]}\left|Q_{i}\right\rangle\left\langle Q_{i}\right|\right)^{-1}\right| X\right\rangle,
\end{aligned}
$$

where $\left|Q_{i}\right\rangle=\Pi_{A B}\left|P_{i}\right\rangle$. Notice that $Q_{i}$ is normalized, since

$$
\sum_{i=1}^{N}\left|Q_{i}\right\rangle=\Pi_{A B} \sum_{i=1}^{N}\left|P_{i}\right\rangle=\Pi_{A B}|I\rangle=|I\rangle,
$$

but in general $Q_{i}$ could not be a POVM because positivity is not preserved by the projection $\Pi_{A B}$. However, we require $Q_{i} \geq 0$ as a condition, whence $\mathbf{Q}$ is a POVM, and the optimal processing is then obtained via the optimal dual of $Q_{i}$.

\section{A. The case of qubits}

The quantum states of a qubit are conveniently represented on the Bloch sphere as follows

$$
\rho=\frac{1}{2}(I+\mathbf{n} \cdot \boldsymbol{\sigma})
$$

where $\boldsymbol{\sigma}=\left(\sigma_{x}, \sigma_{y}, \sigma_{z}\right)$ are the three Pauli operators, and $\mathbf{n}$ is a vector with norm $\|\mathbf{n}\| \leq 1$. Since any positive operator is proportional to a state, any POVM can be represented as follows,

$$
P_{i}=\alpha_{i} I+\beta_{i} \sigma_{x}+\gamma_{i} \sigma_{y}+\delta_{i} \sigma_{z}
$$

where $\left\{\alpha_{i}\right\},\left\{\beta_{i}\right\},\left\{\gamma_{i}\right\}$ and $\left\{\delta_{i}\right\}$ are positive coefficients such that

$$
\beta_{i}^{2}+\gamma_{i}^{2}+\delta_{i}^{2} \leq \alpha_{i}^{2}, \quad \alpha_{i} \geq 0,
$$

and the normalization is given by

$$
\sum_{i=1}^{N} \alpha_{i}=1, \quad \sum_{i=1}^{N} \beta_{i}=\sum_{i=1}^{N} \gamma_{i}=\sum_{i=1}^{N} \delta_{i}=0
$$


Notice that apart from a multiplication factor and a unitary transformation any couple of noncommuting traceless operators $A$ and $B$ is equivalent to the following one

$$
\sigma_{ \pm}(\theta)=\sigma_{x} \cos \theta \pm \sigma_{y} \sin \theta
$$

whose commutator is $i \sigma_{z} \sin 2 \theta$. Therefore, without loss of generality, we will restrict attention to $\sigma_{ \pm}(\theta)$ [26]. Now $\mathcal{S}_{A B}=\operatorname{Span}\left\{\sigma_{x}, \sigma_{y}, I\right\}$, and we consider the case of $\rho_{\mathcal{E}} \in \mathcal{S}_{A B}$. Let us take a general POVM $\mathbf{P}$ such that $\Pi_{\mathcal{S}(\mathbf{P})} \Pi_{\sigma_{x}, \sigma_{y}}=\Pi_{\sigma_{x}, \sigma_{y}}$. By definition, such POVM is $\sigma_{x}, \sigma_{y}$-informationally complete. We can now prove that the operators $\left\{Q_{i}\right\}$ defined by $\left|Q_{i}\right\rangle=\Pi_{\sigma_{x}, \sigma_{y}}\left|P_{i}\right\rangle$ make a POVM. The normalization can be proved as in Eq. (38). On the other hand, $Q_{i}$ is positive, and this can be proved considering Eq. (40). In fact, acting with $\Pi_{\sigma_{x}, \sigma_{y}}$ on $P_{i}$ one has

$$
\Pi_{\sigma_{x}, \sigma_{y}}\left|P_{i}\right\rangle=\left|Q_{i}\right\rangle=\alpha_{i}|I\rangle+\beta_{i}\left|\sigma_{x}\right\rangle+\gamma_{i}\left|\sigma_{y}\right\rangle
$$

Clearly, the conditions for positivity in Eq. (41) are still satisfied. We have then proved that $\left\{Q_{i}\right\}$ is a minimal $\sigma_{x}, \sigma_{y}$-informationally complete POVM. Moreover, since $\rho_{\mathcal{E}} \in \mathcal{S}_{A B}$, then

$$
\operatorname{Tr}\left[P_{i} \rho_{\mathcal{E}}\right]=\left\langle P_{i}\left|\Pi_{\sigma_{x}, \sigma_{y}}\right| \rho_{\mathcal{E}}\right\rangle=\operatorname{Tr}\left[Q_{i} \rho_{\mathcal{E}}\right]
$$

namely $\mathbf{P}$ and $\mathbf{Q}$ give the same probability distribution over the state $\rho_{\mathcal{E}}$, whence they will have the same expectations when averaging over the ensemble $\mathcal{E}$. Therefore, we are in the conditions of Lemma 1, whence for optimal $\mathbf{P}$ the constructed $\mathbf{Q}$ is optimal and minimal.

From now on, we will consider POVMs $\mathbf{P}$ such that $\Pi_{\sigma_{x}, \sigma_{y}}\left|P_{i}\right\rangle=\left|P_{i}\right\rangle$. Moreover, we will restrict our attention to ensembles with a isotropic distribution, having $\rho_{\mathcal{E}}=\frac{I}{2}$. In this case $\pi_{i i}=\operatorname{Tr}\left[P_{i}\right] / 2=\alpha_{i}$. It is clear that we can consider rank one POVMs, since if $P_{i}$ is rank 2 for some $i$, then its spectral decomposition can be written as

$$
P_{i 1}=\frac{\lambda_{2} I}{\lambda_{2}-\lambda_{1}}-\frac{P_{i}}{\lambda_{2}-\lambda_{1}}, P_{i 2}=\frac{\lambda_{1} I}{\lambda_{1}-\lambda_{2}}-\frac{P_{i}}{\lambda_{1}-\lambda_{2}},
$$

where $\lambda_{j}$ are the two eigenvalues of $P_{i}$. The spectral projections belong then to the space $\sigma_{x}, \sigma_{y}$, being linear combinations of $I$ and $P_{i}$. Consequently, any $\sigma_{x}, \sigma_{y^{-}}$ informationally complete POVM can be simulated by a rank one $\sigma_{x}, \sigma_{y}$-informationally complete, whence there exists an optimal minimal rank-one POVM which is $\sigma_{x}, \sigma_{y}$-informationally complete.

Rank-one minimal $\sigma_{x}, \sigma_{y}$-informationally complete POVMs can be easily characterized by restricting the conditions in Eq. (41) as follows

$$
\beta_{i}^{2}+\gamma_{i}^{2}=\alpha_{i}^{2}, \quad \alpha_{i}>0
$$

The matrix $\Lambda \pi^{-1} \Lambda^{\dagger}$ can be written as

$$
\Lambda \pi^{-1} \Lambda^{\dagger}=\sum_{i=1}^{N} \frac{2}{\operatorname{Tr}\left[P_{i}\right]}\left|P_{i}\right\rangle\left\langle P_{i}\right|
$$

which is represented on the orthonormal basis $\left\{\frac{1}{\sqrt{2}}|I\rangle, \frac{1}{\sqrt{2}}\left|\sigma_{x}\right\rangle, \frac{1}{\sqrt{2}}\left|\sigma_{y}\right\rangle\right\}$ in the block-diagonal form

$$
\Lambda \pi^{-1} \Lambda^{\dagger}=\left(\begin{array}{cc}
2 & 0 \\
0 & K
\end{array}\right)
$$

with $K$ being the $2 \times 2$ matrix

$$
K=2\left(\begin{array}{ccc}
\sum_{i=1}^{N} \frac{\beta_{i}^{2}}{\alpha_{i}} & \sum_{i=1}^{N} \frac{\beta_{i} \gamma_{i}}{\alpha_{i}} \\
\sum_{i=1}^{N} \frac{\beta_{i} \gamma_{i}}{\alpha_{i}} & \sum_{i=1}^{N} \frac{\gamma_{i}^{2}}{\alpha_{i}}
\end{array}\right)
$$

The inverse can be easily calculated, and is equal to

$$
\left(\Lambda \pi^{-1} \Lambda^{\dagger}\right)^{-1}=\left(\begin{array}{ccc}
\frac{1}{2} & 0 & 0 \\
0 & \frac{2}{D} \sum_{i=1}^{N} \frac{\gamma_{i}^{2}}{\alpha_{i}} & -\frac{2}{D} \sum_{i=1}^{N} \frac{\beta_{i} \gamma_{i}}{\alpha_{i}} \\
0 & -\frac{2}{D} \sum_{i=1}^{N} \frac{\beta_{i} \gamma_{i}}{\alpha_{i}} & \frac{2}{D} \sum_{i=1}^{N} \frac{\beta_{i}^{2}}{\alpha_{i}}
\end{array}\right)
$$

where $D=\operatorname{det}(K)$.

Using this expression we can evaluate the error for $\sigma_{ \pm}(\theta)$

$$
\begin{aligned}
\delta^{2}\left(\sigma_{ \pm}(\theta)\right)= & \cos ^{2} \theta\left(\frac{\Gamma}{D}-\overline{\left\langle\sigma_{x}\right\rangle^{2}}\right)+\sin ^{2} \theta\left(\frac{B}{D}-\overline{\left\langle\sigma_{y}\right\rangle^{2}}\right) \\
& \mp 2 \sin \theta \cos \theta\left(\frac{\Delta}{D}+\overline{\left\langle\sigma_{x}\right\rangle\left\langle\sigma_{y}\right\rangle}\right)
\end{aligned}
$$

where we defined $\Gamma:=2 \sum_{i=1}^{N} \frac{\gamma_{i}^{2}}{\alpha_{i}}, B:=2 \sum_{i=1}^{N} \frac{\beta_{i}^{2}}{\alpha_{i}}$ and $\Delta:=-2 \sum_{i=1}^{N} \frac{\beta_{i} \gamma_{i}}{\alpha_{i}}$, and consequently $D=B \Gamma-\Delta^{2}$. by

The total error $\delta_{\mathcal{E}}^{2}(\theta):=\delta_{\mathcal{E}}^{2}\left(\sigma_{+}(\theta)\right)+\delta_{\mathcal{E}}^{2}\left(\sigma_{-}(\theta)\right)$ is given

$$
\delta_{\mathcal{E}}^{2}(\theta)=2\left[\cos ^{2} \theta\left(\frac{\Gamma}{D}-\overline{\left\langle\sigma_{x}\right\rangle^{2}}\right)+\sin ^{2} \theta\left(\frac{B}{D}-\overline{\left\langle\sigma_{y}\right\rangle^{2}}\right)\right],
$$

and we can prove by the following argument that the optimal POVM is such that $\Delta=0$. Indeed, consider a POVM $\mathbf{P}$ with given coefficients $\alpha_{i}, \beta_{i}, \gamma_{i}$ corresponding to given values for $B, \Gamma, \Delta$. Now consider the POVM $\mathbf{P}^{\prime}$ with the same coefficients $\alpha_{i}^{\prime}=\alpha_{i}$ and $\beta_{i}^{\prime}=\beta_{i}$ as $\mathbf{P}$ and with $\gamma_{i}^{\prime}=-\gamma_{i}$, corresponding to $B^{\prime}=B, \Gamma^{\prime}=$ $\Gamma^{\prime}$ and $\Delta^{\prime}=-\Delta$. If we take now the POVM $\mathbf{P}^{\prime \prime}:=$ $\frac{1}{2}\left(P_{1}, \ldots, P_{N}, P_{1}^{\prime}, \ldots, P_{N}^{\prime}\right)$, then the corresponding values can be readily calculated to be $B^{\prime \prime}=B=B^{\prime}, \Gamma^{\prime \prime}=\Gamma=$ $\Gamma^{\prime}$ and $\Delta^{\prime \prime}=0$. Correspondingly, the expression for the determinant $D^{\prime \prime}$ becomes $D^{\prime \prime}=B \Gamma \geq D=B \Gamma-\Delta^{2}$. Since the POVM $\mathbf{P}^{\prime \prime}$ can be constructed from any POVM $\mathbf{P}$, then clearly the optimal POVM minimizing the total noise $\delta_{\mathcal{E}}^{2}(\theta)$ is such that $\Delta=0$.

Then, we have that $\left(\Lambda \pi^{-1} \Lambda^{\dagger}\right)^{-1}$ becomes diagonal

$$
\left(\Lambda \pi^{-1} \Lambda^{\dagger}\right)^{-1}=\left(\begin{array}{ccc}
\frac{1}{2} & 0 & 0 \\
0 & \frac{1}{B} & 0 \\
0 & 0 & \frac{1}{\Gamma}
\end{array}\right)
$$


For rank-one POVMs, notice that $\sum_{i=1}^{N} \frac{\beta_{i}^{2}+\gamma_{i}^{2}}{\alpha_{i}}=1$, namely, $B+\Gamma=2$, and the total error is given by

$$
\delta_{\mathcal{E}}^{2}\left(\sigma_{+}(\theta)\right)+\delta_{\mathcal{E}}^{2}\left(\sigma_{-}(\theta)\right)=4\left(\frac{\cos ^{2} \theta}{B}+\frac{1-\cos ^{2} \theta}{2-B}-\frac{\kappa}{2}\right),
$$

with $\kappa=\frac{1}{2}\left(\overline{\left\langle\sigma_{+}(\theta)\right\rangle^{2}} \mathcal{E}+\overline{\left\langle\sigma_{-}(\theta)\right\rangle^{2}}{ }_{\mathcal{E}}\right)$. The minimum of Eq. (55) as a function of $B$ can be easily obtained, leading to the following bound for the total error

$$
\delta_{\mathcal{E}}^{2}\left(\sigma_{+}(\theta)\right)+\delta_{\mathcal{E}}^{2}\left(\sigma_{-}(\theta)\right) \geq 2(1+\sin 2 \theta-\kappa) .
$$

We will now provide two POVMs that achieve the bound. The first one has the following three elements

$$
\begin{aligned}
& P_{1}=p\left(I+\sigma_{x}\right), \\
& P_{2 \pm}=\frac{1-p}{2} I-\frac{p}{2} \sigma_{x} \pm \frac{\sqrt{1-2 p}}{2} \sigma_{y},
\end{aligned}
$$

with $p=\frac{\cos \theta}{2 \cos \theta+\sin \theta}$, and the second one has four elements

$$
\begin{aligned}
& P_{1 \pm}=\frac{p}{2}\left(I \pm \sigma_{x}\right), \\
& P_{2 \pm}=\frac{1-p}{2}\left(I \pm \sigma_{y}\right),
\end{aligned}
$$

with $p=\frac{\cos \theta}{\cos \theta+\sin \theta}$. For equal uncertainties, the minimum product of the r.m.s. errors is given by

$$
\sqrt{\delta_{\mathcal{E}}^{2}\left(\sigma_{+}(\theta)\right)} \sqrt{\delta_{\mathcal{E}}^{2}\left(\sigma_{-}(\theta)\right)}=\delta_{\mathcal{E}}^{2}\left(\sigma_{ \pm}(\theta)\right)=1+\sin 2 \theta-\kappa .
$$

We recall that the results of the qubit case from Eq. (46) to Eq. (59) are obtained under the assumptions of isotropic ensemble $\rho_{\mathcal{E}}=\frac{I}{2}$. In fact, we want to stress that even in the qubit case, whenever $\rho_{\mathcal{E}}$ corresponding to the prior ensemble is not fully lying in the space $\sigma_{x}, \sigma_{y}$, it is not proved that the optimal POVM is $\sigma_{x}, \sigma_{y^{-}}$ informationally complete.

\section{CONCLUSIONS}

In this paper we have introduced the concept of $A B$ informationally complete measurements, within the context of Quantum indirect estimation theory. Compared with a customary infocomplete measurements, the $A B$ infocomplete one in principle allows a less noisy joint estimation of all the moments of two noncompatible observables $A$ and $B$. The concept of $A B$ can be also easily extended to more than two observable, but we have not analyzed such generalization. We solved the case of qubits, showing that a $\sigma_{x} \sigma_{y}$-infocomplete measurement is less noisy than any infocomplete one. The relation between the concept of $A B$-infocompleteness and the notion of joint measurement of observables $A$ and $B$ has also been discussed. The relation between minimality and optimality of $A B$-infocomplete measurements remains an open problem.
[1] P. Busch, Int. J. Th. Phys. 30, 1217 (1991).

[2] E. Prugovečki, Int. J. Th. Phys. 16, 321 (1977).

[3] C. A. Fuchs, quant-ph/0205039

[4] C. M. Caves, C. A. Fuchs, and R. Schack, J. Math. Phys. 43, 4537 (2002).

[5] K. E. Cahill and R. J. Glauber, Phys. Rev. 177, 1857 (1969).

[6] G. M. D'Ariano, M. G. A. Paris, and M. F. Sacchi, Advances in Imaging and Electron Physics 128, 205-308 (2003).

[7] A. S. Holevo, Probabilistic and Statistical Aspects of Quantum Theory (North Holland, Amsterdam, 1982).

[8] E. B. Davies, Quantum Theory of Open Systems, Academic Press, New York, 1976.

[9] P. Busch, M. Grabowski, and P. Lahti, Operational Quantum Physics, Lecture Notes in Physics 31 (Springer, Berlin, 1995).

[10] F. E. Schroeck, Quantum Mechanics on Phase Space (Kluwer, Dordrecht, 1996).

[11] V. Peřinova, A. Lukš, and J. Peřina, Phase in Optics (World Scientific, Singapore, 1998).

[12] G. M. D'Ariano, P. Perinotti, and M. F. Sacchi, J. Opt. B 6, S487 (2004); G. M. D'Ariano, P. Perinotti, and M. F. Sacchi, in "Proc. 8th Int. Conf. on Squeezed States and Uncertainty Relations", ed. by H. Moya-Cessa et al.,p. 86 (Rinton, Princeton, 2003), also available at quant-ph/0309161.

[13] D. M. Appleby, H. B. Dang, and C. A. Fuchs, quantph/0707.2071, and references therein.

[14] G. M. D'Ariano, P. Perinotti, and M. F. Sacchi, Phys. Rev. A 72, 042108 (2005).

[15] G. M. D'Ariano and P. Perinotti, Phys. Rev. Lett. 98, 020403 (2007).

[16] R. J. Duffin and A. C. Schaeffer, Trans. Amer. Math. Soc. 72, 341 (1952).

[17] P. G. Casazza, Taiw. J. Math. 4, 129 (2000).

[18] S. Li, Numer. Funct. Anal. Optim. 16, 1181 (1995).

[19] R. B. Bhapat, Linear Algebra and Linear Models, (Springer-Verlag, New York, 2000).

[20] G. M. D'Ariano and P. Perinotti, inQuantum Commutation, Measurement and Computing, ed. by O. Hirota. J. H. Shapiro, and M. Sasaki, NICT press (Tokyo, 2007), pag. 327

[21] F.Buscemi, G. M. D’Ariano, M. Keyl, P. Perinotti, and R. F. Werner, Clean Positive Operator Valued Measures J. Math. Phys. 46082109 (2005)

[22] E. Arthurs and J. L. Kelly, Bell. Syst. Tech. J. 44, 725 (1965)

[23] A. M. Perelomov, Comm. Math. Phys. 26, 222 (1972).

[24] F. Zhang, Matrix Theory. Basic Results and Techniques, 
(Springer-Verlag, New York, 1999).

[25] The proof that $\Pi_{A B}\left(\Lambda \pi^{-1} \Lambda^{\dagger}\right)^{-1} \Pi_{A B} \geq$ $\left(\Pi_{A B} \Lambda \pi^{-1} \Lambda^{\dagger} \Pi_{A B}\right)^{-1}$ is the following. Consider two positive invertible operators $X$ and $Y$ such that $X \geq Y$. Then we have $Y^{-\frac{1}{2}} X Y^{-\frac{1}{2}} \geq I$, and consequently $\left(Y^{-\frac{1}{2}} X Y^{-\frac{1}{2}}\right)^{-1}=Y^{\frac{1}{2}} X^{-1} Y^{\frac{1}{2}} \leq I$, and finally $X^{-1} \leq Y^{-1}$. Now one can prove [24] that for a general invertible positive operator $X$ and any projection $\Pi$ one has $\Pi X^{-1} \Pi=\left(\Pi X \Pi-\Pi X[(I-\Pi) X(I-\Pi)]^{-1} X \Pi\right)^{-1}$, where the inverse $[(I-\Pi) X(I-\Pi)]^{-1}$ is on the support of $I-\Pi$. Now, clearly

$$
\Pi X \Pi-\Pi X[(I-\Pi) X(I-\Pi)]^{-1} X \Pi \leq \Pi X \Pi,
$$

and consequently $\Pi X^{-1} \Pi \geq(\Pi X \Pi)^{-1}$.

[26] Notice that it is irrelevant to add a trace to $A$ and $B$, since this can be done by adding an operator proportional to the identity, e. g. $X^{\prime}=X+k I$, and the minimum error in the estimation of $\left\langle X^{\prime}\right\rangle$ would be

$$
\delta_{\mathcal{E}}^{2}\left(X^{\prime}\right)=\sum_{i=1}^{N}\left(c_{i}^{X}+k\right)^{2} \operatorname{Tr}\left[\rho_{\mathcal{E}} P_{i}\right]-\left({\overline{\langle X\rangle_{\mathcal{E}}}}+k\right)^{2}=\delta_{\mathcal{E}}^{2}(X)
$$

since the processing function of the identity for the optimal processing is $c_{i}^{I}=1$. 